\title{
EMMPRIN Modulates Epithelial Barrier Function through a MMP-Mediated Occludin Cleavage
}

\section{Implications in Dry Eye Disease}

\author{
Eric Huet, ${ }^{* \dagger}$ Benoit Vallée, ${ }^{*}$ Jean Delbé, ${ }^{* \dagger}$ \\ Samia Mourah, ${ }^{\ddagger}$ Virginie Prulière-Escabasse, ${ }^{\S}$ \\ Magali Tremouilleres, ${ }^{\dagger}$ Kenji Kadomatsu, ${ }^{\natural}$ \\ Serge Doan, " Christophe Baudouin, ${ }^{\| * *}$ \\ Suzanne Menashi, ${ }^{*}$ and Eric E. Gabison ${ }^{\| * * \dagger \dagger}$ \\ From the CRRET Laboratory," Université Paris-Est, CNRS, Créteil, \\ France; the Department of Bioengineering, ${ }^{\dagger}$ Institute of \\ Technology, Université Paris-Est, Créteil, France; the Laboratory \\ of Pharmacology, ${ }^{\ddagger}$ AP-HP, Hospital Saint-Louis, Paris, France, \\ INSERM U 955, ${ }^{\S}$ Université Paris-Est, CNRS, Créteil, France; the \\ Department of Biochemistry, "Nagoya University Graduate \\ School of Medicine, Nagoya, Japan; Fondation A de Rothschild, \\ Paris, France; and INSERM UMR S 968,** and the Institut de la \\ Vision, Paris, France; Université Paris $7,{ }^{\text {t† }}$ Diderot, Paris, France
}

Dry eye is a common disease that develops as a result of alteration of tear fluid, leading to osmotic stress and a perturbed epithelial barrier. Matrix metalloproteinase-9 (MMP-9) may be important in dry eye disease, as its genetic knockout conferred resistance to the epithelial disruption. We show that extracellular matrix metalloproteinase inducer (EMMPRIN; also termed CD147), an inducer of MMP expression, participates in the pathogenesis of dry eye through MMPmediated cleavage of occludin, an important component of tight junctions. EMMPRIN expression was increased on the ocular surface of dry eye patients and correlated with those of MMP-9. High osmolarity in cell culture, mimicking dry eye conditions, increased both EMMPRIN and MMP-9 and resulted in the disruption of epithelial junctions through the cleavage of occludin. Exogenously added recombinant EMMPRIN had similar effects that were abrogated in the presence of the MMP inhibitor marimastat. Membrane occludin immunostaining was markedly increased in the apical corneal epithelium of both EMMPRIN and MMP-9 knock-out mice. Furthermore, an inverse correlation between EMMPRIN and occludin membrane staining was consistently observed both in vitro and in vivo as a function of corneal epithelial cells differentiation. These data suggest a possible role of EMMPRIN in regulating the amount of occludin at the cell surface in homeostasis beyond pathological situations such as dry eye disease, and EMMPRIN may be essential for the formation and maintenance of organized epithelial structure. (Am J Pathol 2011, 179:1278-1286; DOI: 10.1016/j.ajpath.2011.05.036)

Epithelial cells interact to form a polarized tissue that acts as a protective barrier against infection and environmental stress while allowing exchange of molecules with the extracellular surroundings. Such a continuous permeability barrier functions through the formation of tight junctions that mediate adhesion between adjacent epithelial cells. ${ }^{1}$ Tight junctions are composed of transmembrane proteins, including occludin, claudin, and a number of peripheral membrane structural proteins, such as zonula occludens (ZO), and are associated with a variety of regulatory proteins. ${ }^{2}$ Disruption of these junctions is thought to perturb the normal cellular environment and lead to numerous pathological situations such as dry eye, asthma, and inflammatory bowel disease. ${ }^{3}$

Dry eye is a common disease that develops as a result of quantitative or qualitative reduction in tear fluid, which normally serves as a source of hydration to the ocular surface. ${ }^{4}$ This can be caused by decreased tear production or increased tear evaporation. The resultant high osmolarity in the tear film leads to disruption in the intercellular connections and is considered to be a key factor in the pathogenesis of dry eye syndrome. ${ }^{5}$ Matrix metalloproteinase-9 (MMP-9) was suggested to be of central importance in the disruption of corneal epithelium under increased osmolarity. ${ }^{6}$ It was shown to be able to cleave

Supported in part by the Fondation de L'Avenir, study ET8-489, and Fondation Dalloz, Institut de France (E.E.G.)

Accepted for publication May 31, 2011.

Address reprint requests to Suzanne Menashi, Ph.D., CRRET Laboratory, Université Paris-Est Créteil, 61 av du Gal De Gaulle, 94010 Creteil Cedex, France. E-mail: menashi@u-pec.fr. 
the tight junction protein occludin. ${ }^{6}$ Furthermore, MMP-9 knockout mice were shown to be resistant to experimental desiccating stress, as they have demonstrated reduced alteration of epithelial barrier compared with wildtype mice. ${ }^{6}$ Increased MMP-9 was observed in an experimental murine model of dry eye, in which it was associated with alteration in corneal surface regularity and epithelial barrier function. ${ }^{7-11}$ Increased MMP-9 levels were also observed in the tear fluid of patients with dry

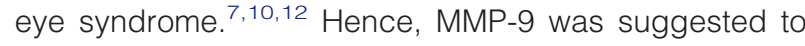
be responsible for the cellular responses in dry eye syndrome, but the factors behind its deregulated production have not been determined.

Extracellular matrix metalloproteinase inducer (EMMPRIN; also termed CD147) is the major and most characterized cell surface regulator of MMP production. ${ }^{13,14}$ EMMPRIN was first identified in tumor cells, where it is particularly enriched. However, it is also expressed in nontumoral tissues, in particular in normal epithelia such as skin, lung, or cornea, and may be implicated in numerous physiological and pathological systems where proteolysis occurs. EMMPRIN has been shown to induce the expression of a variety of MMPs, including MMP-9, without an effect on their physiological inhibitors, TIMP-1 and TIMP-2, thereby increasing the net proteolytic potential.

In this study, we demonstrate a role for EMMPRIN in the disruption of the epithelial integrity observed in dry eye syndrome, an excellent model for studying epithelial barrier function. Increased EMMPRIN expression by high osmolarity resulted in an MMP-9-mediated loss of cell surface occludin and cell-cell contact. The decrease in EMMPRIN levels in dry eye patients after treatment and improvement of clinical symptoms further supports the role of EMMPRIN in the function of the epithelial barrier in vivo. Moreover, the inverse distribution of EMMPRIN and occludin observed as a function of differentiation of epithelial cells, both in culture and in the stratified corneal epithelium in vivo, suggests that the regulation of occludin by EMMPRIN represents a novel physiological function.

\section{Materials and Methods}

\section{Antibodies and Chemicals}

Antibodies and chemicals used in this study were as follows: anti-EMMPRIN mAb: anti-CD147, clone HIM6 (Becton Dickinson France, Le Pont de Claix, France); anti-occludin pAb: anti-occludin N-term (Invitrogen, Cergy Pontoise, France); anti-GAPDH: clone 6C5 (Applied Biosystems, Courtabouef, France); horseradishperoxidase-conjugated anti-mouse antibody (Jackson Immuno Research, Immunotech, Marseille, France); antimouse IgG Alexa 488 and anti-rabbit IgG Alexa 594 (Invitrogen); Marimastat (British Biotechnology, Oxford, UK); BM Chemi-luminescence Western Blotting Substrate (POD) and DAPI (Roche Diagnostics, Meylan, France); and Protease Inhibitor Cocktail Set V EDTA-free (Calbiochem, VWR International, Strasbourg, France).

\section{Cells and Tissues}

SV40-immortalized human corneal epithelial cells (Dr. Kaoru Araki-Sasaki, Riken Cell Bank, Saitama, Japan) were cultured in Dulbecco's modified Eagle's medium and Ham's nutrient mixture F12 (1:1) culture medium (Invitrogen), supplemented with $1 \%(\mathrm{v} / \mathrm{v})$ antibiotic/antimycotic solution, $2 \mathrm{mmol} / \mathrm{L}$ L-glutamine, $5 \mu \mathrm{g} / \mathrm{mL}$ insulin, $10 \mathrm{ng} / \mathrm{mL}$ human epidermal growth factor (SHEM medium) and $10 \%(\mathrm{v} / \mathrm{v})$ fetal calf serum (FCS; PAA Laboratories, Les Mureaux, France). For immunohistochemistry studies in mice corneas, eyes were dissected from wild type (WT), EMMPRIN and MMP-9 knock-out (KO) mice ${ }^{15,16}$ and were embedded in optimal cutting temperature (OCT) compound (VWR International) before sectioning and immunostaining for occludin and EMMPRIN.

\section{Cell Treatments}

Corneal epithelial cells were cultured in SHEM medium containing 10\% serum and complemented with $2.5 \mathrm{mmol} / \mathrm{L}$ $\mathrm{CaCl}_{2}$. When cells reached approximately $90 \%$ confluence, culture medium was replaced with fresh SHEM medium with added $2.5 \mathrm{mmol} / \mathrm{L} \mathrm{CaCl}_{2}$ but not containing serum, and 0,70 , or $90 \mathrm{mmol} / \mathrm{L} \mathrm{NaCl}$ was then added to increase the osmolarity (corresponding to 312,450 , or $500 \mathrm{mOsM}$, respectively). Cells were incubated for 24 hours before protein extraction and conditioned medium collection. To study epithelial cell differentiation in vitro, cells were cultured in SHEM medium with or without added calcium.

\section{Measurements of Transepithelial Electrical Resistance}

Corneal epithelial cells were seeded in the upper chambers of a Transwell tissue culture plates (Costar, 12-mm diameter, 0.4- $\mu \mathrm{m}$ pore size, Corning Life Sciences, VWR) in SHEM medium containing 10\% FCS and allowed to reach confluence. Cells were then cultured for a further 2 days in SHEM medium containing 2\% FCS and 2.5 $\mathrm{mmol} / \mathrm{L} \mathrm{CaCl}$, after which the transepithelial electrical resistance (TER) was measured with an epithelial voltohmmeter (Evom, World Precision Instruments, Sarasota, FL). When TER reached $100 \Omega \mathrm{cm}^{2}$ or greater, cells were treated with either $90 \mathrm{mmol} / \mathrm{L} \mathrm{NaCl}$ or $10 \mu \mathrm{g} / \mathrm{mL}$ recEMMRPIN for 24 hours, and measurements were repeated.

\section{Expression and Purification of Recombinant EMMPRIN}

Soluble recombinant EMMPRIN (recEMMPRIN), consisting of the extracellular domain of human EMMPRIN, was generated after truncation of its transmembrane and cytoplasmic parts at $\mathrm{LeU}_{200}$ by PCR-directed mutagenesis and cloning into pcDNA3 vector. This was transfected to $\mathrm{CHO}$ cells, and positive clones with stable expression of soluble recombinant EMMPRIN were identified by Western blot. $\mathrm{CHO}$-conditioned medium was purified first on a Concanavalin A-sepharose column (GE Health Care, Velisy, France) equilibrated and washed with $0.5 \mathrm{~mol} / \mathrm{L}$ 
$\mathrm{NaCl}$ in $20 \mathrm{mmol} / \mathrm{L}$ Tris- $\mathrm{HCl}, \mathrm{pH}$ 7.4. Elution was carried out with $0.2 \mathrm{~mol} / \mathrm{L}$ methyl- $\alpha$-D-mannopyranoside (SigmaAldrich, St. Quentin Fallavier, France). Eluted fraction was dialyzed against $0.1 \mathrm{~mol} / \mathrm{L} \mathrm{NaCl}$ in $20 \mathrm{mmol} / \mathrm{L}$ Tris- $\mathrm{HCl}$, $\mathrm{pH} 7.4$, and then applied to a 1-mL Mono Q 5/50 column (GE Health Care) at $0.5 \mathrm{~mL} / \mathrm{min}$ previously equilibrated with $0.1 \mathrm{~mol} / \mathrm{L} \mathrm{NaCl}$ in $20 \mathrm{mmol} / \mathrm{L}$ Tris- $\mathrm{HCl}, \mathrm{pH}$ 7.4. The column was washed using the same buffer until the $A^{280}$ dropped to $<1 \%$ of its maximum. Protein bound to the column were eluted with a linear gradient of $\mathrm{NaCl}$ increasing from $0.1 \mathrm{~mol} / \mathrm{L}$ to $1 \mathrm{~mol} / \mathrm{L}$ in $20 \mathrm{mmol} / \mathrm{L}$ Tris- $\mathrm{HCl}$, $\mathrm{pH} 7.4$, buffer. Purity of EMMPRIN in each chromatographic step was evaluated by sodium dodecyl sulfatepolyacrylamide gel electrophoresis (SDS-PAGE) and confirmed by Western blot.

\section{Triton X-114 Extraction of Membrane Proteins}

After treatment with recEMMPRIN, cells were washed and lysed with ice-cold 2\% Triton X-114 in Tris-buffered saline (TBS; $50 \mathrm{mmol} / \mathrm{L}$ Tris- $\mathrm{HCl}, \mathrm{pH} 7.4$, and $150 \mathrm{mmol} / \mathrm{L}$ $\mathrm{NaCl}$ ). After centrifugation of the lysate at $10,000 \mathrm{~g}$ for 10 minutes to remove the Triton-insoluble material, the supernatant was partitioned into detergent (lower) and aqueous (upper) phases by incubation at $37^{\circ} \mathrm{C}$ for 1 minute, followed by centrifugation at $1000 \mathrm{~g}$ for 5 minutes. After protein determination of the aqueous phase, fractions of the detergent phase containing identical protein amounts were assayed by Western blotting.

\section{Western Blot}

Membranes were immunoblotted with either anti-EMMPRIN mAb or occludin pAb overnight at $4^{\circ} \mathrm{C}$ or with antiGAPDH mAb for 1 hour at room temperature, followed by enhanced chemiluminescence detection of bound primary antibodies as previously described. ${ }^{17}$ Protein loading was verified by Ponceau Red staining and by comparison with the intensity of GAPDH bands.

\section{Gelatin Zymography}

Conditioned medium collected from cells cultured for 24 hours in serum-free SHEM medium was analyzed for MMP-9 as described previously ${ }^{18}$ on $10 \%$ SDS-PAGE gels containing $1 \mathrm{mg} / \mathrm{mL}$ gelatin.

\section{Quantitative RT-PCR}

Relative expression of EMMPRIN and MMP-9 was determined by real time quantitative PCR using Perfect MasterMix-Probe (Any-Genes, France) on LightCycler 2.0 (Roche Diagnostics) as described previously. ${ }^{19}$ Data were normalized to the $\beta 2$ microglobulin (b2m) housekeeping gene transcripts.

\section{Small Interfering RNA Transfection}

Two different EMMPRIN siRNA (Ambion, Applied Biosystems) or scramble siRNA (BLOCK-iT fluorescent oligo,
Invitrogen) were transfected into cells using oligofectamine transfection reagent in Opti-MEM I (Invitrogen), as described previously. ${ }^{17}$ Cells were then incubated for 18 hours in SHEM medium with 10\% FCS and for a further 24 hours in serum-free SHEM medium with or without $\mathrm{NaCl}$ addition before analysis.

\section{Confocal Immunohistochemistry and Immunocytochemistry}

Cryostat sections $(8-\mu \mathrm{m})$ were prepared from frozen WT and $\mathrm{KO}$ cornea and immunostained after fixation in chilled acetone for 10 minutes, re-hydratation in PBS (Invitrogen), and incubation in blocking solution (3\% BSA in PBS) for 30 minutes. For immunocytochemistry, cells were seeded on glass slides, cultured for 2 to 3 days in the presence of $10 \%$ FCS, and treated as indicated in Figures 2-5. Slides were then washed and fixed in $4 \%$ paraformaldehyde/DMEM $(\mathrm{v} / \mathrm{v})$. Section or cell slides were then incubated for 1 hour with either anti-EMMPRIN mAb or anti-occludin $\mathrm{pAb}$ and then for 30 minutes with affinity-purified secondary antibodies (donkey anti-mouse and/or anti-goat IgG, Alexa 488 and 594, respectively). The slides were mounted and examined with a laser scanning confocal microscope (Leica Lasertechnik, Heidelberg, Germany). In negative controls, the primary antibody was substituted with PBS.

\section{Patients and Clinical Data}

Conjunctival impression cytology specimens were obtained from 15 patients with dry eye syndrome before and after treatment with unpreserved artificial tears (Larmabak, Laboratoire Thea, Clermont-Ferrand, France). The study was conducted in compliance with the Declaration of Helsinki, Scotland amendment, 2000. All patients received specific explanations on impression cytology principles and the study aims and gave informed consent for the procedure and subsequent use of conjunctival specimens. Patients' characteristics are as follows: median age, 53 years (range, 43 to 71 years), sex ratio, 1:1; all Caucasian/white. No statistically significant modification in either Schirmer test or tear break-up time were observed after 6 weeks treatment. The Schirmer test value was $5.3 \mathrm{~mm}( \pm 2.3)$ before and 5.9 $\mathrm{mm}( \pm 3.0)$ after treatment and tear film break-up time 4.5 seconds ( \pm 2.3 ) before and 4.8 seconds ( \pm 3.1 ) after treatment. Eight of 15 patients reported improved symptoms, seven of 15 reported unchanged symptoms, and none were worse after treatment, $P=0.265$ by paired $t$-test. Specimens were collected in the superior bulbar conjunctiva by using 0.20- $\mu \mathrm{m}$ polyether sulfone filters (Supor Membranes, Gelman Sciences, Ann Arbor, MI) after instillation of 1 drop of $0.4 \%$ oxybuprocain. Membranes were later placed in RNA (Sigma-Aldrich) and stored at $-20^{\circ} \mathrm{C}$ before quantitative RT-PCR analysis.

\section{Statistical Analysis}

Data are represented as mean \pm SEM. The Wilcoxon test was used to analyze the significance of the change in EMMPRIN and MMP-9 (between groups) on treatment. The 
Spearman rank correlation was used to evaluate the association between the differences in mRNA levels in EMMPRIN and MMP-9 normalized to the reference gene b2m on treatment in a 15 patients' samples. The Student test was used to analyze the significance of the change in EMMPRIN and MMP-9 mRNA or protein levels after cell treatment.

\section{Results}

\section{Correlation between EMMPRIN and MMP-9 Levels in Dry Eye Patients}

MMP-9 RNA was previously reported to be increased in tears of dry eye patients ${ }^{7}$ and was suggested to be directly associated with the disruption of epithelial barrier function. To assess the potential role of EMMPRIN in MMP-9 increase, conjunctival impression cytology was performed in 15 patients diagnosed with dry eye syndrome before treatment and 1 month after treatment, with preservative-free physiological saline solution. This treatment is known to reduce osmolarity and to improve symptoms. ${ }^{20-22}$ EMMPRIN and MMP-9 gene expression was evaluated by quantitative RT-PCR on the cells attached to the filters as described. ${ }^{23}$ A statistically significant reduction in both MMP-9 and EMMPRIN mRNA levels (Wilcoxon test, $P=0.0026$ and $P=0.0014$, respectively) was observed after treatment (Figure 1). The reduction in EMMPRIN mRNA was correlated with that of MMP-9 $(P<$ 0.05 , Spearman rank correlation coefficient $=0.6393, \mathrm{df}=$ 13), suggesting that the increase of MMP-9 in dry eye patients may be a consequence of EMMPRIN increase.

\section{Recombinant EMMPRIN Induces MMP-9 in Human Corneal Epithelial Cells: Consequences in Epithelial Barrier Function}

To explore the possible role of EMMPRIN in the disruption of the epithelial barrier observed in dry eye patients, we looked at the effect of exogenously added recombinant EMMPRIN (recEMMPRIN) on the expression of MMP-9 and on the tight junction component occludin in cultured corneal epithelial cells.

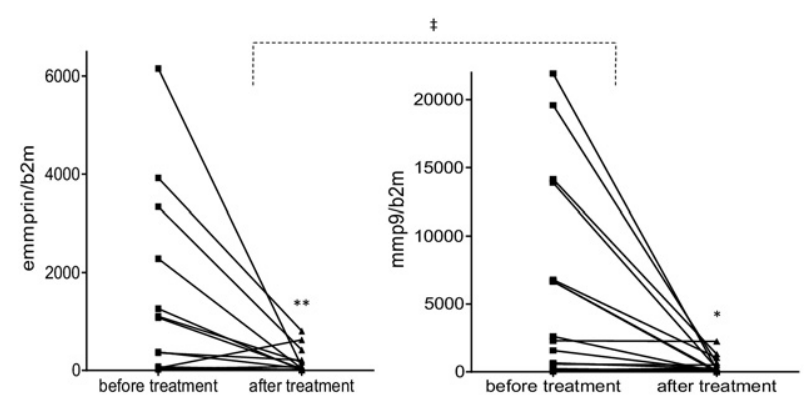

Figure 1. Evaluation of EMMPRIN and MMP-9 mRNA levels in dry eye patients before and after treatment with artificial tears. Conjunctival impression cytology samples were subjected to real-time PCR analysis. Wilcoxon $\left({ }^{*} P<0.05\right.$ and $\left.{ }^{* *} P<0.01\right)$ and Spearman $\left({ }^{\ddagger} P<0.05\right)$ rank test analysis were performed. In each case, the number of copies of EMMPRIN and MMP-9 was normalized to the reference gene $\beta 2$ microglobulin (b2m).
A recEMMPRIN $(\mu \mathrm{g} / \mathrm{mL})$
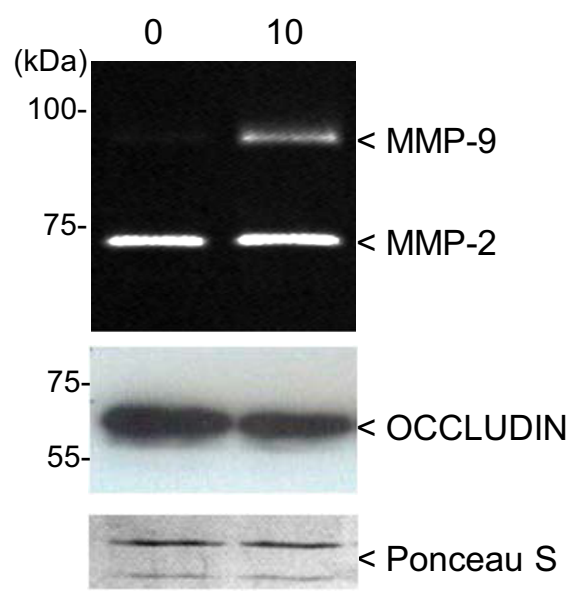

B
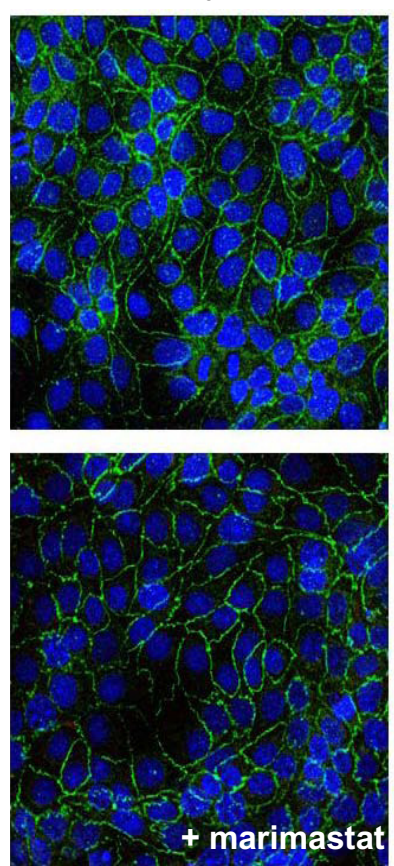
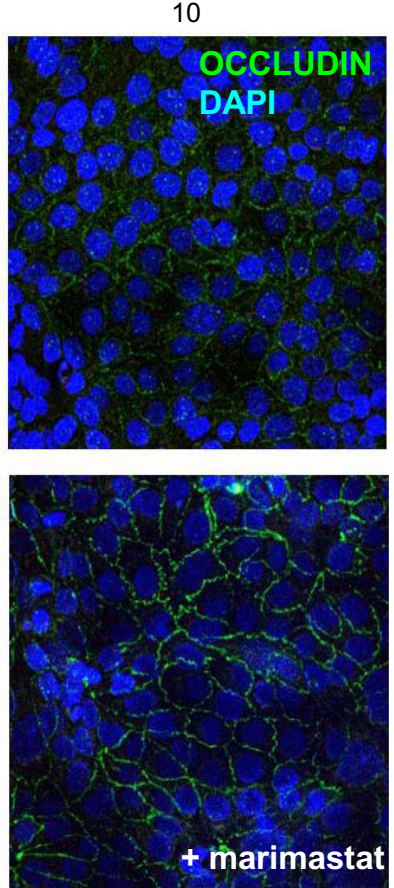

Figure 2. EMMPRIN induction of MMP-9 is associated with a decreased membrane expression of occludin in human corneal epithelial cells. Corneal epithelial cells were treated with $10 \mu \mathrm{g} / \mathrm{mL}$ recombinant EMMPRIN (recEMMPRIN) in serum-free medium for 24 hours. A: Gelatin zymography was performed on the conditioned medium and Western blot on the Triton X-114 cell extract. EMMPRIN treatment induced MMP-9 and caused a decreased in occludin protein levels. Ponceau $S$ was used as loading control. B: Confocal immunocytochemistry of the treated cells reveals a decrease in the cell surface staining of occludin. This EMMPRIN's effect on occludin was abrogated when the cells were simultaneously treated with $10 \mu \mathrm{mol} / \mathrm{L}$ marimastat.

Gelatin zymography of the conditioned medium obtained from corneal epithelial cells treated with exogenously added recEMMPRIN (10 $\mu \mathrm{g} / \mathrm{mL})$ demonstrated an increase in MMP-9 production (Figure 2A). This was associated with a decrease in occludin protein levels as shown by Western blot of cell lysates (Figure 2A). Similarly, a decrease in the cell surface expression of occludin was observed by confocal immunohistochemistry after recEMMPRIN treatment 
(Figure 2B). The co-addition of the MMPs inhibitor marimastat $(10 \mu \mathrm{mol} / \mathrm{L})$ with recEMMPRIN abrogated EMMPRIN's effect on occludin surface expression (Figure 2B,). This suggests that MMP activity is necessary for the EMMPRINmediated occludin disappearance and hence the disruption of epithelial cell-cell junctions.

\section{High Osmolarity Increases EMMPRIN}

\section{Expression in Human Corneal Epithelial Cells} and Is Associated with an Increase in MMP-9 and a Loss of Epithelial Cell-Cell Junctions

The question of the possible role of EMMPRIN in dry eye was addressed using high osmolarity in culture, a commonly used cellular model which mimics the pathogenesis of dry eye. ${ }^{24}$ Normal osmolarity of culture medium, 312 mOsM, was switched to hyperosmolar media, 450 and $500 \mathrm{mOsM}$, by adding, respectively, 70 and 90 $\mathrm{mmol} / \mathrm{L} \mathrm{NaCl}$. Although tear hyperosmolarity in patients with dry eye rarely reaches $360 \mathrm{mOsM}$, most in vitro studies of hyperosmolarity use higher levels (400 to 600 $\mathrm{mOsM}$ ) to demonstrate response. ${ }^{25}$ Figure 3 shows that the addition of $90 \mathrm{mmol} / \mathrm{L} \mathrm{NaCl}$ to corneal epithelial cells had the greatest effect in increasing EMMPRIN expression at both the protein and RNA levels (Figure 3, A and C). Although a smaller increase was observed with 70 $\mathrm{mmol} / \mathrm{L} \mathrm{NaCl}$, no effect could be discerned at the lower concentrations (30 and $50 \mathrm{mmol} / \mathrm{L} \mathrm{NaCl}$; not shown).

Gelatin zymography analysis of serum-free conditioned medium of corneal epithelial cells only shows single bands of MMP-9 and MMP-2 with no detectable additional bands of the active gelatinases. However, the mere detection of the MMP-9 protein in biological samples is generally considered to represent the existence of MMP-9-mediated proteolysis. The MMP-9 band increased in the presence of increasing $\mathrm{NaCl}$ concentrations, parallel to the increase in EMMPRIN (Figure 3B). MMP-9 RNA levels were also significantly increased in both $70 \mathrm{mmol} / \mathrm{L}$ and $90 \mathrm{mmol} / \mathrm{L} \mathrm{NaCl}$ (Figure 3D). No effect of high osmolarity could be observed on either TIMP-1 or TIMP-2 production by reverse zymography (data not shown).

The increased EMMPRIN level after exposure to higher osmolarity was also shown by confocal immunohistochemistry (Figure 3E, red). Conversely, the strong occludin staining observed at the intercellular junctions of untreated corneal epithelial cells decreased in the hyperosmolar-treated cells (Figure 3E, green). This was associated with increased intercellular spaces observed by bright field microscopic observations when cells were incubated with $90 \mathrm{mmol} / \mathrm{L} \mathrm{NaCl}$ (Figure 3E, BF), confirming the disruptive effect of high osmolarity on the epithelial cell barrier. TER measurements confirm this change in epithelial permeability. TER of $122 \pm 12 \Omega \mathrm{cm}^{2}$ was observed for an untreated monolayer of epithelial cells that dropped to $75 \pm 13 \Omega \mathrm{cm}^{2}$ with $90 \mathrm{mmol} / \mathrm{L} \mathrm{NaCl}$ treatment and to $86 \pm 7 \Omega \mathrm{cm}^{2}$ with $10 \mu \mathrm{g} / \mathrm{mL}$ of recEMMPRIN treatment (all values represent the mean \pm $\mathrm{SD}$ of two experiments conducted in triplicate).
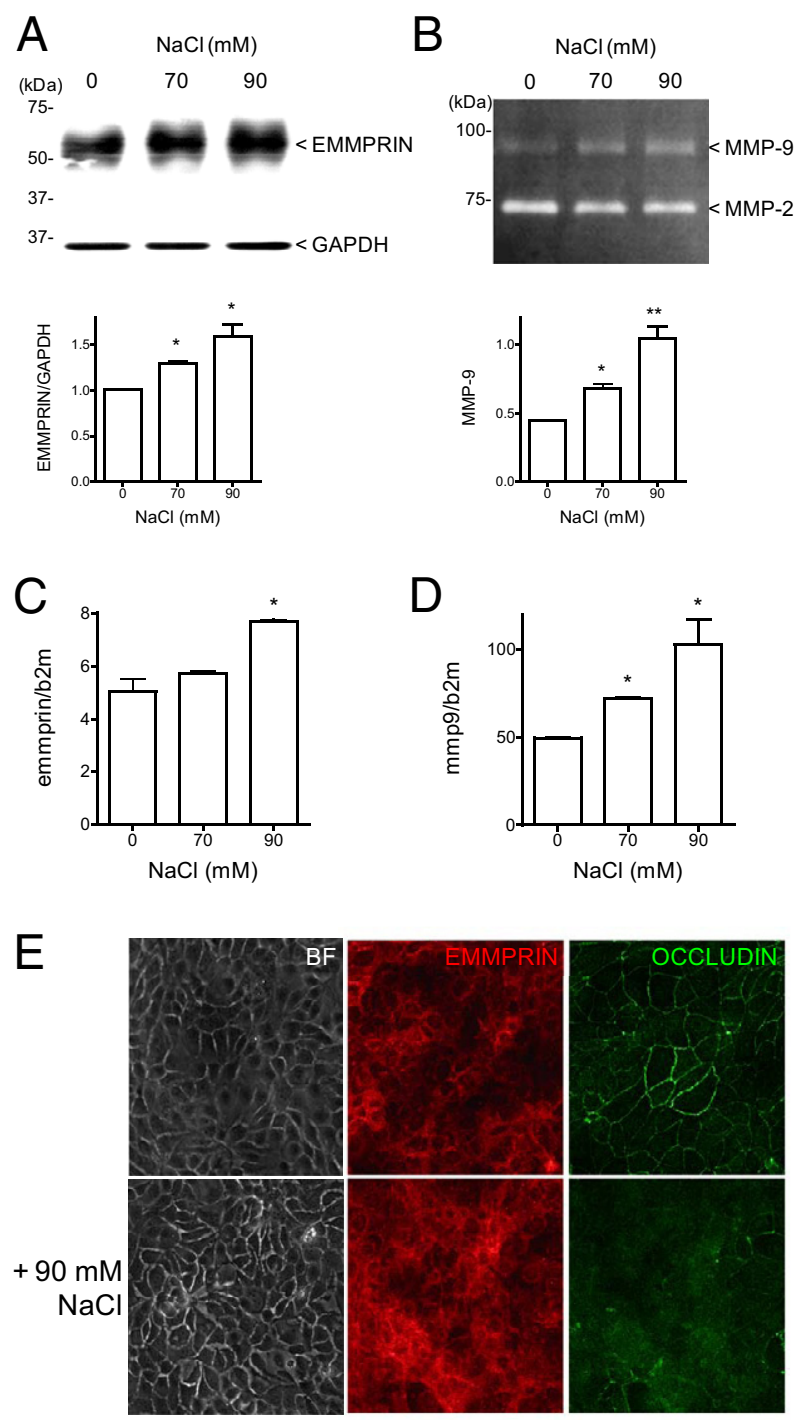

Figure 3. High osmolarity increases EMMPRIN expression in human corneal epithelial cells and is associated with an increased of MMP-9 and a loss of occludin and epithelial cell-cell junctions. When corneal epithelial cells reached about $90 \%$ of confluence, culture medium was then replaced with fresh serum free medium supplemented with 70 or $90 \mathrm{mmol} / \mathrm{L} \mathrm{NaCl}$ to increase osmolarity. After 24 hours' incubation, cell lyzates were subjected to EMMPRIN analysis by Western blot (A), and condition medium was analyzed by gelatin zymography for MMP-9 (B). EMMPRIN (C) and MMP-9 (D) mRNA were quantified by real time PCR 4 hours after $\mathrm{NaCl}$ addition. ${ }^{*} P<0.05$. The consequences of high osmolarity on epithelial cell barrier function were analyzed by bright field and confocal microscopy (E). Increased intercellular spaces can be noted in high osmolarity conditions by bright field (BF) microscopy. Double labeled confocal immunostaining revealed and increased in EMMPRIN (red) and a decrease in occludin membrane staining (green) in high osmolarity conditions $(+90 \mathrm{mmol} / \mathrm{L} \mathrm{NaCl})$.

\section{EMMPRIN Inhibition Suppresses MMP-9 Induction by High Osmolarity and Restores Occludin Membranous Expression}

EMMPRIN siRNA transfection of corneal epithelial cells abrogated high osmolarity-mediated induction of both EMMPRIN and MMP-9 levels, as shown by Western blot and gelatin zymography, respectively, as compared with the scrambled control siRNA transfected cells (Figure $4 A)$. Similar results were obtained using two different 
A
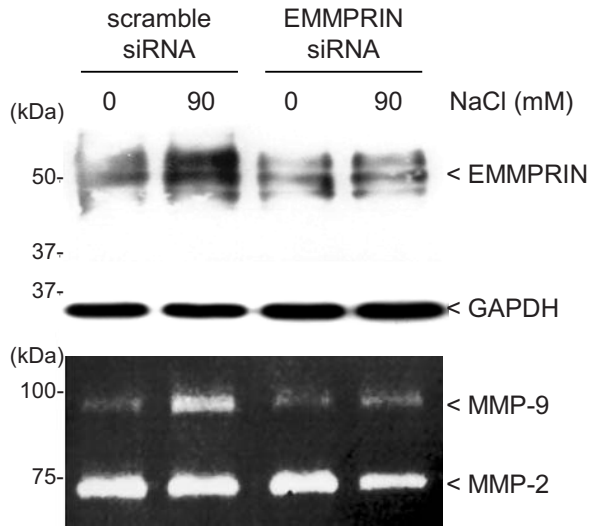

B scramble siRNA
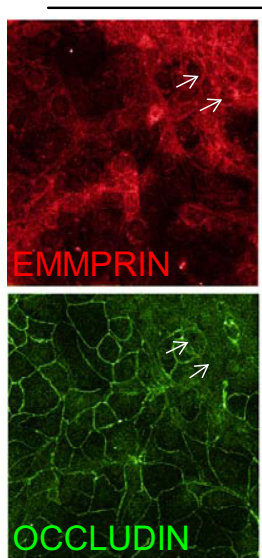
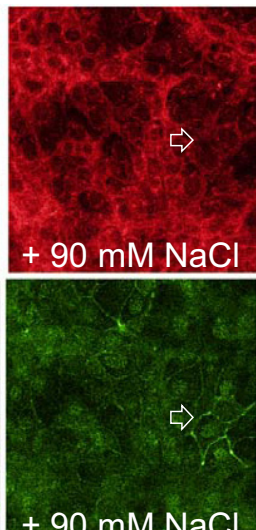

$+90 \mathrm{mM} \cdot \mathrm{NaCl}$
EMMPRIN SiRNA
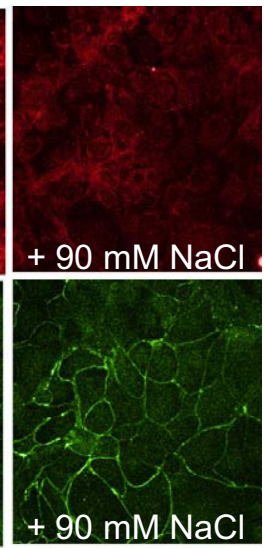

Figure 4. EMMPRIN inhibition suppresses MMP-9 induction by high osmolarity and restores occludin membranous expression. A: EMMPRIN siRNA transfection of corneal epithelial cells inhibited high osmolarity mediated induction of EMMPRIN shown by Western blot and of MMP-9 shown by gelatin zymography. B: Double-labeled confocal immunocytochemistry analysis revealed that the specific inhibition of EMMPRIN by siRNA transfection of corneal epithelial cells restored membrane occludin staining in high osmolarity conditions $(+90 \mathrm{mmol} / \mathrm{L}$ $\mathrm{NaCl}$, not observed with scramble siRNA (EMMPRIN in red, occludin in green). Dissociated expression of EMMPRIN and occludin is indicated by arrows, where areas of high (lined arrows) and low (open arrows) EMMPRIN expression correspond respectively to low and high membrane occludin.

EMMPRIN siRNA. Furthermore, double-labeled immunocytochemistry showed that the inhibition of EMMPRIN by siRNA also restored membrane occludin staining in highosmolarity conditions (Figure 4B). Of note, a detailed examination of the immunostaining images revealed that areas of higher EMMPRIN staining corresponded to low occludin membranous staining (arrows), underscoring a dissociation of the surface expression of the two molecules within the same cell. We reasoned, therefore, that a regulation mechanism may exist between EMMPRIN and occludin expression, even under homeostasis conditions.

\section{Dissociation of EMMPRIN and Occludin Membranous Expression during Epithelial Cell Differentiation/Stratification in Vitro and in Vivo}

We have previously shown that EMMPRIN levels are highest in the basal layers of the corneal epithelium, gradually de- creasing from basal to the superficial cells. ${ }^{18}$ Interestingly, occludin levels have been reported to be the highest in the apical layers of the corneal epithelium. ${ }^{6}$ This prompted us to look whether the role of EMMPRIN in regulating occludin in dry eye conditions can exist under physiological conditions.

We first looked for a differential expression of EMMPRIN and occludin during calcium mediated epithelial cell differentiation in vitro. Modulation of calcium concentration in the culture medium is commonly used to study epithelial cell differentiation and stratification. Figure 5 shows that increasing calcium concentrations was followed by a decrease in membranous EMMPRIN expression and an increase in that of occludin, supporting the possible role of EMMPRIN in the modulation of occludin membranous expression in corneal epithelial cells.

The stratified epithelium of the cornea represents an in vivo model of differentiation, as the basal layers are known to contain the undifferentiated or "stem" cells, whereas the terminally differentiated epithelial cells reside in the apical surface. The potential role of EMMPRIN in the regulation of occludin expression in epithelial stratification in vivo was also examined on corneas obtained from wild-type (WT) and knock-out (KO) mice for EMMPRIN. Corneas from MMP-9 KO mice were examined in parallel to evaluate the role for the MMP-9 inducing activity of EMMPRIN in epithelial physiology. Occludin immunostaining in cornea sections from these mice is shown in Figure 6A. The most noticeable difference can be observed in the apical epithelium, where a more pronounced occludin membrane staining in the form of thick lines is seen in both EMMPRIN and MMP-9 KO mice while being hardly noticeable in the WT mice. Double immunostaining of wild-type corneas visualized with confocal microscopy confirmed the inverse relationship between EMMPRIN and occludin membrane expression throughout the epithelium (Figure 6B). EMMPRIN staining was highest in the basal epithelial cells and gradually decreased toward the superficial cells (Figure 6B, red). By contrast, although occludin staining was detectable throughout the epithelium, membranous occludin was observed only in the most superficial layer in the form of dots or short thin lines, whereas occludin staining, which was also observed in the deeper

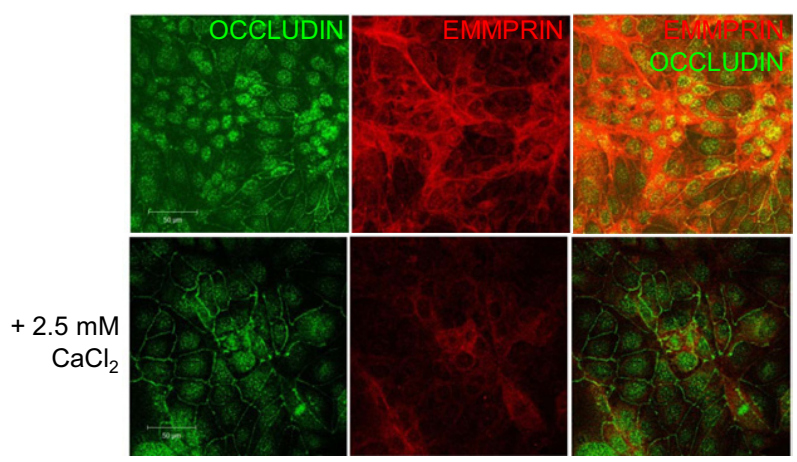

Figure 5. Modulation of EMMPRIN and occludin expression in corneal epithelial cells following calcium mediated differentiation/stratification in vitro. Double labeled confocal immunocytochemistry of EMMPRIN (red) and occludin (green) before (upper panel) and after (lower panel) calcium supplementation $\left(+2.5 \mathrm{mmol} / \mathrm{L} \mathrm{CaCl}_{2}\right)$ in the culture medium. A decrease in membranous EMMPRIN expression and an increase in that of occludin are present in higher calcium concentration. Bar $=50 \mu \mathrm{m}$. 


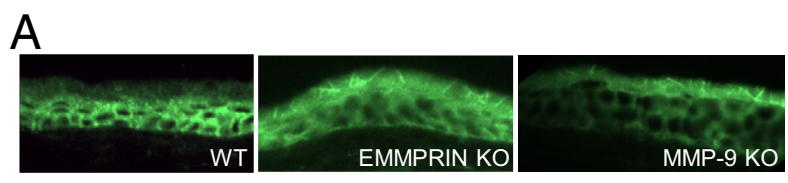

B
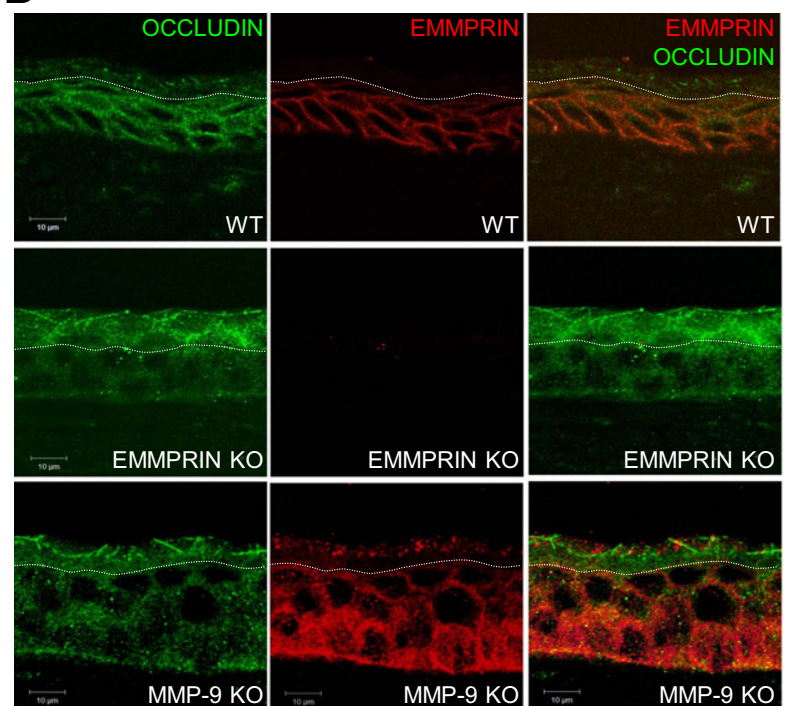

Figure 6. EMMPRIN and occludin expression in corneal epithelium of wildtype, EMMPRIN, and MMP-9 KO mice. A: Immunohistochemical analysis of occludin (green) expression revealed different pattern of expression between the WT and the KO corneal epithelium. In both EMMPRIN and MMP-9 KO, stronger apical occludin membrane staining appearing as longer and thicker lines than in the WT epithelium. B: Double labeled confocal immunohistochemistry of EMMPRIN and occludin in WT and KO mice. In WT mice, EMMPRIN (red) is mainly expressed in the basal layers, gradually decreasing toward the apical layers where its expression is barely detected. The typical membranous staining of occludin (green) is only observed in the apical areas where EMMPRIN staining is absent. Occludin membranous staining is much more pronounced in both EMMPRIN and MMP-9 KO mice and appears in deeper apical layers of the epithelium as longer and thicker lines. Note that the white dotted line was drawn at the point where occludin staining becomes membranous. Below this line occludin staining appears to be mainly cytoplasmic. In WT corneas, this line also corresponds to the extinction of EMMPRIN staining. Above this line, the expression of EMMPRIN occasional detected as is shown in the MMP-9 KO panel, was dissociated from the membranous occludin staining.

layers, appeared to be mainly cytoplasmic (Figure 6B, green). In EMMPRIN KO mice, the absence of EMMPRIN was associated with a marked increased occludin membrane staining in the apical layers, appearing as much longer and thicker lines that covered more cell membrane area. An increase in occludin staining was observed in the MMP-9 KO mice that was similar to that in the EMMPRIN KO epithelium, further suggesting that the regulation of MMP-9 may be behind the EMMPRIN-mediated effect on occludin in corneal epithelium. However, the implication of other proteases induced by EMMPRIN during differentiation cannot be excluded. Altogether, these results support the role of EMMPRIN in mediating occludin membranous expression at least partially via MMP-9.

\section{Discussion}

The barrier function of the epithelium is critical for the maintenance and regulation of its permeability characteristics, and depends on the integrity of the tight junctions.
By cleaving occludin, MMP-9 has been shown to be able to disrupt the corneal epithelial barrier and to contribute to the pathogenesis of dry eye syndrome. $6,7,10-12,26$ However, the factors responsible for the up-regulation of MMP-9 in this pathological condition are not fully understood.

Our work presented here suggests that EMMPRIN, by inducing MMP-9, is an important actor in the regulation of epithelium integrity, both in corneal physiology and in dry eye disease through its action on cell surface occludin. This was demonstrated both by the exogenous addition of recombinant EMMPRIN to cultured epithelial cells and by the increased endogenous expression of EMMPRIN in these cells by high osmolarity, conditions used to mimic the dry eye situation in vitro. These treatments resulted in an MMP-9 increase and a concomitant disappearance of occludin. The fact that the MMP inhibitor marimastat protected occludin from EMMPRIN's effect suggests that the loss of occludin from epithelial cell surface involved an MMP-dependent cleavage. Our findings thus describe a new role for EMMPRIN as a regulator of tight junction structure and integrity through its MMP-inducing function. Furthermore, the increase in MMP-9 levels observed in vivo in the ocular surface of dry eye patients was correlated with EMMPRIN levels, and both were markedly decreased after treatment, supporting the role of EMMPRIN behind the MMP increase.

We have previously shown that EMMPRIN expression in the normal cornea and skin presented a gradient with high levels in the basal undifferentiated epithelial cells which decrease toward the superficial layers of the more differentiated cells. ${ }^{18}$ The fact that occludin, on the contrary, is known to be localized in the apical layers of the stratified epithelium led us to hypothesize that this inverse distribution observed in the normal epithelium may also result from an EMMPRIN-mediated decrease in occludin. This possibility, implying a role for EMMPRIN in the regulation of the epithelial barrier under physiological conditions, was questioned both in vitro and in vivo. We first used an in vitro model of epithelial cell differentiation through the modulation of calcium concentrations. ${ }^{27-30}$ On differentiation/stratification, the typical membranous staining of EMMPRIN was markedly reduced and only diffusely localized in the cytoplasm, concomitantly with the appearance of clear membranous occludin staining. This striking inverse correlation, which was repeatedly observed between EMMPRIN decreased expression and the appearance of membranous occludin, thus evokes a more general pattern of regulation beyond pathological situations such as in dry eye disease.

In vivo studies using EMMPRIN KO mice revealed that when EMMPRIN is absent in the corneal epithelium, cell surface occludin, which is normally limited to the superficial layer, has shown both an increase in level of expression, appearing as thicker and longer lines on the membrane, and a localization in deeper epithelial layers. A similar occludin distribution was observed in the corneal epithelium of MMP-9 KO mice, further supporting an MMP-dependent action of EMMPRIN in the regulation of occludin cellular expression and localization in the stratified epithelium. Interestingly, Pflugfelder et al. demon- 
strated that MMP-9 KO were more resistant to superficial epithelial cell desquamation under desiccating stress conditions, ${ }^{6}$ possibly owing to the increased membranous occludin at this localization. The normal gradient in EMMPRIN expression, decreasing from the basal to the apical epithelial layers, may thus represent a possible protection mechanism allowing occludin membranous expression at the corneal apical surface and the maintenance of tight junction integrity in physiological conditions. However, proteolysis induced through EMMPRIN action may not be the only mechanism involved in the physiological regulation of occludin expression in the epithelium, as the lack of EMMPRIN or MMP-9 does not abolish the occludin gradient. Other mechanisms, such as regulation of gene expression or membrane trafficking, may also contribute to the formation of the occludin gradient.

Although the role of MMP-9 in the pathogenesis of dry eye through occludin cleavage has already been suggested, its role in the physiological expression of occludin remains to be determined. MMP-9 has been reported both in the apical and in the basal surface of the epithelium, depending on whether the protein, RNA, or activity was evaluated. Although constitutive protein expression of MMP-9 has been reported in the superficial layers of normal cornea, which greatly increased when challenged with desiccating stress, ${ }^{31}$ in situ gelatin zymography on untreated cornea localized a stronger gelatinolytic activity in the basal layer of the epithelium, increasing throughout the epithelium in dry eye conditions. ${ }^{32}$ Furthermore, an in situ hybridization technique revealed that MMP-9 RNA, undetected in normal epithelium, is induced after injury and sustained during epithelial stratification within the basal layer, ${ }^{33}$ suggesting that MMP-9 is produced essentially by the basal cells where EMMPRIN is localized. A growing body of evidence has indicated that cancer progression is associated with decreased tight junctions, and the loss of occludin is generally correlated with tumor development. ${ }^{34,35}$ It has also largely demonstrated that EMMPRIN expression is greatly increased in cancer tissues and is correlated with progression and, in some cases, with poor prognosis. ${ }^{36,37}$ In view of our results presented here, it is tempting to speculate that EMMPRIN induction during tumor progression may lead to an MMP-dependent cleavage of occludin and may decrease cell contact, thus promoting invasion. However, whether other factors beside MMPs contribute to the inverse relationship between EMMPRIN and occludin in cancer cells remains to be investigated. We have recently shown that EMMPRIN increases hypoxia-inducible factors (HIF 1 and 2) in both endothelial cells and in melanoma tumor cells. ${ }^{38}$ These transcription factors were shown to down-regulate occludin and to disrupt tight junction, ${ }^{39}$ thus representing an additional potential mechanism for the regulation of occludin by EMMPRIN in cancer cells.

On the basis of the results obtained in this study, the following scenario may be postulated on the possible events that follow increased tear osmolarity. The increased EMMPRIN expression induces MMPs, including MMP-9, which then cleaves occludin and disrupts the tight junctions. The released MMPs may favor the shedding of EMMPRIN from the epithelial cell surface. ${ }^{40}$ Soluble EMMPRIN, which has previously been reported in tear fluids, ${ }^{41}$ can then further induce both EMMPRIN and MMP production, creating a perpetuating loop that contributes to the disruption of the epithelial barrier and aggravates disease severity. Our ongoing studies investigate the possible use of EMMPRIN as a biological marker for dry eye severity.

By controlling occludin levels on the epithelial surface, EMMPRIN may play a crucial role in the formation and maintenance of organized epithelial structures. The identification of this new molecular mechanism underlying disruption of tight junctions will facilitate the development of novel treatments for diseases in which tight junction barriers are compromised.

\section{Acknowledgments}

We thank Patricia Zadigu and Celine Narayanane for valuable technical help.

\section{References}

1. Yoshida $Y$, Ban $Y$, Kinoshita S: Tight junction transmembrane protein claudin subtype expression and distribution in human corneal and conjunctival epithelium. Invest Ophthalmol Vis Sci 2009, 50:21032108

2. Langbein L, Grund C, Kuhn C, Praetzel S, Kartenbeck J, Brandner JM, Moll I, Franke WW: Tight junctions and compositionally related junctional structures in mammalian stratified epithelia and cell cultures derived therefrom. Eur J Cell Biol 2002, 81:419-435

3. Vermeer PD, Denker J, Estin M, Moninger TO, Keshavjee S, Karp P Kline JN, Zabner J: MMP9 modulates tight junction integrity and cell viability in human airway epithelia. Am J Physiol Lung Cell Mol Physiol 2009, 296:L751-L762

4. Pflugfelder SC, Solomon A, Stern ME: The diagnosis and management of dry eye: a twenty-five-year review. Cornea 2000, 19:644-649

5. Sullivan BD, Whitmer D, Nichols KK, Tomlinson A, Foulks GN, Geerling G, Pepose JS, Kosheleff V, Porreco A, Lemp MA: An objective approach to dry eye disease severity. Invest Ophthalmol Vis Sci 2010, 51:6125-6130

6. Pflugfelder SC, Farley W, Luo L, Chen LZ, de Paiva CS, Olmos LC, Li $D Q$, Fini ME: Matrix metalloproteinase-9 knockout confers resistance to corneal epithelial barrier disruption in experimental dry eye. Am J Pathol 2005, 166:61-71

7. Chotikavanich S, de Paiva CS, Li de Q, Chen JJ, Bian F, Farley WJ Pflugfelder SC: Production and activity of matrix metalloproteinase-9 on the ocular surface increase in dysfunctional tear syndrome. Invest Ophthalmol Vis Sci 2009, 50:3203-3209

8. Corrales RM, Stern ME, De Paiva CS, Welch J, Li DQ, Pflugfelder SC Desiccating stress stimulates expression of matrix metalloproteinases by the corneal epithelium. Invest Ophthalmol Vis Sci 2006, 47:32933302

9. De Paiva CS, Corrales RM, Villarreal AL, Farley WJ, Li DQ, Stern ME Pflugfelder SC: Corticosteroid and doxycycline suppress MMP-9 and inflammatory cytokine expression. MAPK activation in the corneal epithelium in experimental dry eye, Exp Eye Res 2006, 83:526-535

10. Luo L, Li DQ, Corrales RM, Pflugfelder SC: Hyperosmolar saline is a proinflammatory stress on the mouse ocular surface. Eye Contact Lens 2005, 31:186-193

11. Smith VA, Rishmawi $H$, Hussein $H$, Easty DL: Tear film MMP accumulation and corneal disease. Br J Ophthalmol 2001, 85:147-153

12. Acera A, Rocha G, Vecino E, Lema I, Duran JA: Inflammatory markers in the tears of patients with ocular surface disease. Ophthalmic Res 2008, 40:315-321 
13. Gabison EE, Hoang-Xuan T, Mauviel A, Menashi S: EMMPRIN/ CD147, an MMP modulator in cancer, development and tissue repair. Biochimie 2005, 87:361-368

14. Huet E, Gabison EE, Mourah S, Menashi S: Role of emmprin/CD147 in tissue remodeling. Connect Tissue Res 2008, 49:175-179

15. Chen S, Kadomatsu K, Kondo M, Toyama Y, Toshimori K, Ueno S, Miyake $\mathrm{Y}$, Muramatsu T: Effects of flanking genes on the phenotypes of mice deficient in basigin/CD147. Biochem Biophys Res Commun 2004, 324:147-153

16. Liu Z, Zhou X, Shapiro SD, Shipley JM, Twining SS, Diaz LA, Senior RM, Werb Z: The serpin alpha1-proteinase inhibitor is a critical substrate for gelatinase B/MMP-9 in vivo. Cell 2000, 102:647-655

17. Huet E, Vallee B, Szul D, Verrecchia F, Mourah S, Jester JV, Hoang Xuan T, Menashi S, Gabison EE: Extracellular matrix metalloproteinase inducer/CD147 promotes myofibroblast differentiation by inducing alpha-smooth muscle actin expression and collagen gel contraction: implications in tissue remodeling. FASEB J 2008, 22: 1144-1154

18. Gabison EE, Mourah S, Steinfels E, Yan L, Hoang-Xuan T, Watsky MA, De Wever B, Calvo F, Mauviel A, Menashi S: Differential expression of extracellular matrix metalloproteinase inducer (CD147) in normal and ulcerated corneas: role in epithelio-stromal interactions and matrix metalloproteinase induction. Am J Pathol 2005, 166:209-219

19. Gabison EE, Chastang P, Menashi S, Mourah S, Doan S, Oster M, Mauviel A, Hoang-Xuan T: Late corneal perforation after photorefractive keratectomy associated with topical diclofenac: involvement of matrix metalloproteinases. Ophthalmology 2003, 110:1626-1631

20. Methodologies to diagnose and monitor dry eye disease: report of the Diagnostic Methodology Subcommittee of the International Dry Eye WorkShop: (2007). Ocul Surf 2007, 5:108-152

21. Baudouin C: A new approach for better comprehension of diseases of the ocular surface. J Fr Ophtalmol 2007, 30:239-246

22. Suzuki M, Massingale ML, Ye F, Godbold J, Elfassy T, Vallabhajosyula M, Asbell PA: Tear osmolarity as a biomarker for dry eye disease severity. Invest Ophthalmol Vis Sci 2010, 51:4557-4561

23. Argueso P, Balaram M, Spurr-Michaud S, Keutmann HT, Dana MR, Gipson IK: Decreased levels of the goblet cell mucin MUC5AC in tears of patients with Sjogren syndrome. Invest Ophthalmol Vis Sci 2002, 43:1004-1011

24. Li DQ, Chen Z, Song XJ, Luo L, Pflugfelder SC: Stimulation of matrix metalloproteinases by hyperosmolarity via a JNK pathway in human corneal epithelial cells. Invest Ophthalmol Vis Sci 2004, 45:43024311

25. Liu H, Begley C, Chen M, Bradley A, Bonanno J, McNamara NA, Nelson JD, Simpson T: A link between tear instability and hyperosmolarity in dry eye. Invest Ophthalmol Vis Sci 2009, 50:3671-3679

26. Li DQ, Pflugfelder SC: Matrix metalloproteinases in corneal inflammation. Ocul Surf 2005, 3:S198-S202

27. Argueso P, Tisdale A, Spurr-Michaud S, Sumiyoshi M, Gipson IK: Mucin characteristics of human corneal-limbal epithelial cells that exclude the rose bengal anionic dye. Invest Ophthalmol Vis Sci 2006 47:113-119

28. Brandner JM, Kief S, Grund C, Rendl M, Houdek P, Kuhn C, Tschachler E, Franke WW, Moll I: Organization and formation of the tight junction system in human epidermis and cultured keratinocytes. Eur J Cell Biol 2002, 81:253-263

29. Breitkreutz D, Stark HJ, Plein P, Baur M, Fusenig NE: Differential modulation of epidermal keratinization in immortalized (HaCaT) and tumorigenic human skin keratinocytes (HaCaT-ras) by retinoic acid and extracellular Ca2+. Differentiation 1993, 54:201-217

30. Heenen M, De Graef C, Galand P: Kinetics of the calcium induced stratification of human keratinocytes in vitro. Cell Prolif 1992, 25:233-240

31. De Paiva CS, Pangelinan SB, Chang E, Yoon KC, Farley WJ, Li DQ, Pflugfelder SC: Essential role for C-Jun N-terminal kinase 2 in corneal epithelial response to desiccating stress. Arch Ophthalmol 2009 127:1625-1631

32. De Paiva CS, Yoon KC, Pangelinan SB, Pham S, Puthenparambil LM, Chuang EY, Farley WJ, Stern ME, Li DQ, Pflugfelder SC: Cleavage of functional IL-2 receptor alpha chain (CD25) from murine corneal and conjunctival epithelia by MMP-9. J Inflamm (Lond) 2009, 6:31

33. Ye HQ, Azar DT: Expression of gelatinases A and B, and TIMPs 1 and 2 during corneal wound healing. Invest Ophthalmol Vis Sci 1998, 39:913-921

34. Tsukita S, Yamazaki Y, Katsuno T, Tamura A, Tsukita S: Tight junctionbased epithelial microenvironment and cell proliferation. Oncogene 2008, 27:6930-6938

35. Weber CR, Nalle SC, Tretiakova M, Rubin DT, Turner JR: Claudin-1 and claudin-2 expression is elevated in inflammatory bowel disease and may contribute to early neoplastic transformation. Lab Invest 2008, 88:1110-1120

36. Buergy D, Fuchs T, Kambakamba P, Mudduluru G, Maurer G, Post S, Tang Y, Nakada MT, Yan L, Allgayer H: Prognostic impact of extracellular matrix metalloprotease inducer: immunohistochemical analyses of colorectal tumors and immunocytochemical screening of disseminated tumor cells in bone marrow from patients with gastrointestinal cancer. Cancer 2009, 115:4667-4678

37. Li Y, Xu J, Chen L, Zhong WD, Zhang Z, Mi L, Zhang Y, Liao CG, Bian HJ, Jiang JL, Yang XM, Li XY, Fan CM, Zhu P, Fu L, Chen ZN: HAb18G (CD147), a cancer-associated biomarker and its role in cancer detection. Histopathology 2009, 54:677-687

38. Bougatef F, Quemener C, Kellouche S, Naimi B, Podgorniak MP Millot G, Gabison EE, Calvo F, Dosquet C, Lebbe C, Menashi S, Mourah S: EMMPRIN promotes angiogenesis through hypoxia-inducible factor-2alpha-mediated regulation of soluble VEGF isoforms and their receptor VEGFR-2. Blood 2009, 114:5547-5556

39. Harten SK, Shukla D, Barod R, Hergovich A, Balda MS, Matter K, Esteban MA, Maxwell PH: Regulation of renal epithelial tight junctions by the von Hippel-Lindau tumor suppressor gene involves occludin and claudin 1 and is independent of E-cadherin. Mol Biol Cell 2009, 20:1089-1101

40. Egawa N, Koshikawa N, Tomari T, Nabeshima K, Isobe T, Seiki M: Membrane type 1 matrix metalloproteinase (MT1-MMP/MMP-14) cleaves and releases a 22-kDa extracellular matrix metalloproteinase inducer (EMMPRIN) fragment from tumor cells. J Biol Chem 2006 , 281:37576-37585

41. Maatta M, Tervahartiala T, Kaarniranta K, Tang Y, Yan L, Tuukkanen J, Sorsa T: Immunolocalization of EMMPRIN (CD147) in the human eye and detection of soluble form of EMMPRIN in ocular fluids. Curr Eye Res 2006, 31:917-924 